\title{
The influence of non-stoichiometry on the switching kinetics of strontium-titanate ReRAM devices
}

K. Fleck, N. Aslam, S. Hoffmann-Eifert, V. Longo, F. Roozeboom, W. M. M. Kessels, U. Böttger, R. Waser, and S. Menzel

Citation: J. Appl. Phys. 120, 244502 (2016); doi: 10.1063/1.4972833

View online: http://dx.doi.org/10.1063/1.4972833

View Table of Contents: http://aip.scitation.org/toc/jap/120/24

Published by the American Institute of Physics

\section{Articles you may be interested in}

Atomic layer deposition of $\mathrm{HfO} 2$ using $\mathrm{HfCp}(\mathrm{NMe} 2) 3$ and $\mathrm{O} 2$ plasma

J. Appl. Phys. 35, 01B13001B130 (2016); 10.1116/1.4972210

Vertical power MOS transistor as a thermoelectric quasi-nanowire device

J. Appl. Phys. 120, 244903244903 (2016); 10.1063/1.4973275

Multi-bit dark state memory: Double quantum dot as an electronic quantum memory

J. Appl. Phys. 120, 244301244301 (2016); 10.1063/1.4972340

Planar composite chiral metamaterial with broadband dispersionless polarization rotation and high transmission J. Appl. Phys. 120, 245102245102 (2016); 10.1063/1.4972977 


\title{
The influence of non-stoichiometry on the switching kinetics of strontium-titanate ReRAM devices
}

\author{
K. Fleck, ${ }^{1, \text { a) }}$ N. Aslam, ${ }^{2}$ S. Hoffmann-Eifert, ${ }^{2, b)}$ V. Longo, ${ }^{3, c)}$ F. Roozeboom, ${ }^{3, d)}$ \\ W. M. M. Kessels, ${ }^{3}$ U. Böttger, ${ }^{1, b)}$ R. Waser, ${ }^{1, e)}$ and S. Menzel ${ }^{2, b)}$ \\ ${ }^{1}$ Institute of Materials in Electrical Engineering and Information Technology II, RWTH Aachen University, \\ Aachen 52074, Germany \\ ${ }^{2}$ Peter Grünberg Institute, Forschungszentrum Jülich GmbH, 52425 Jülich, Germany \\ ${ }^{3}$ Department of Applied Physics, Eindhoven University of Technology, 5600 MB Eindhoven, The Netherlands
}

(Received 19 August 2016; accepted 8 December 2016; published online 30 December 2016)

\begin{abstract}
Compared to conventional NAND flash resistive switching metal-oxide cells show a number of advantages, like an increased endurance, lower energy consumption, and superior switching speed. Understanding the role of defects for the resistive switching phenomenon in metal oxides is crucial for their improvement and thereby also for their acceptance as a next generation data storage device. Strontium titanate (STO) is considered a model material due to its thoroughly investigated defect chemistry. This paper presents a comparative study of the switching kinetics for three different compositions $[\mathrm{Sr}] /([\mathrm{Sr}]+[\mathrm{Ti}])$ of 0.57 (Sr-rich), 0.50 (stoichiometric STO), and 0.46 (Ti-rich STO). The STO films, deposited by atomic layer deposition, were integrated in Pt/STO/TiN nanocrossbars with a feature size of $100 \mathrm{~nm}$. By analysis of the transient currents, the switching kinetics are investigated between $10 \mathrm{~ns}$ and $10^{4} \mathrm{~s}$ for the SET and $10 \mathrm{~ns}$ and $100 \mathrm{~s}$ for the RESET. A clear influence of the composition on the degree of nonlinearity of the switching kinetics was observed. Applying an analytical model for the oxygen vacancy migration, we were able to explain the differences in the SET kinetics by composition-dependent changes in the thermal conductivity and by a lower activation energy for the Ti-rich sample. This might be utilized in design rules of future ReRAM devices. Published by AIP Publishing. [http://dx.doi.org/10.1063/1.4972833]
\end{abstract}

\section{INTRODUCTION}

Due to the increasing importance of information technology, the amount of data in the world is growing at an ever rising pace. This development drives the search for new nonvolatile memory technologies with fast write and read operations, high endurance and good data retention. The redox based resistive memory (ReRAM) is among the most promising concepts currently under discussion. ${ }^{1-3}$ ReRAM cells basically consist of a metal-oxide-metal structure (Fig. 1(a)). Typical materials involved would be either binary $\left(\mathrm{Ta}_{2} \mathrm{O}_{5}\right.$, $\mathrm{TiO}_{2}$, and $\left.\mathrm{HfO}_{2}\right)$ or ternary $\left(\mathrm{SrTiO}_{3}\right)$ transition metal oxides as the resistive switching layer and metals like $\mathrm{Ti}, \mathrm{Ta}, \mathrm{W}, \mathrm{Pt}$ or conductive TiN, ITO, or Nb: $\mathrm{SrTiO}_{3}$ for the electrodes. ${ }^{3,4}$ The information is stored in the form of the resistive state of the memory cell. This can be either a high resistive state (HRS) and a low resistive state (LRS) or several multilevel states in between those two extrema. ${ }^{5}$ Through the application of an appropriate voltage signal, the reversible switching between

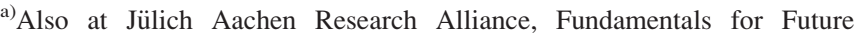
Information Technology, Jülich 52425, Germany. Electronic mail: fleck@ iwe.rwth-aachen.de.

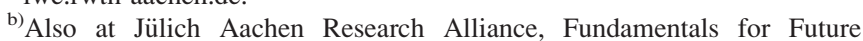
Information Technology, Jülich 52425, Germany.

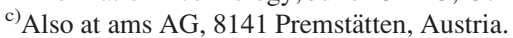

d) Also at Thin Film Technologies Department at TNO, High Tech Campus 21, 5656 AE Eindhoven, The Netherlands.

e)Also at Jülich Aachen Research Alliance, Fundamentals for Future Information Technology, Jülich 52425, Germany and Grünberg Institut, Forschungszentrum Jülich GmbH, 52425 Jülich, Germany.
}

the states can be induced. As the $\mathrm{Pt} / \mathrm{Sr}_{1+x} \mathrm{Ti}_{1+y} \mathrm{O}_{3+(x+2 y)} / \mathrm{TiN}$ cells used in this work belong to the bipolar switching valence change mechanism (VCM) type of ReRAM, the process of setting the cell from the HRS to the LRS requires a voltage $V_{\text {SET }}$ whose polarity is different from $V_{\text {RESET }}$, the voltage used to reset the cell to the HRS. ${ }^{6}$ This bipolar switching behavior stems from the movement of mobile donors (here oxygen vacancies) and the associated redox reaction in the cation sublattice that characterizes the VCM. Most VCM devices require an initial electroforming process before they can be switched repeatedly. During this step, oxygen is locally removed from a)

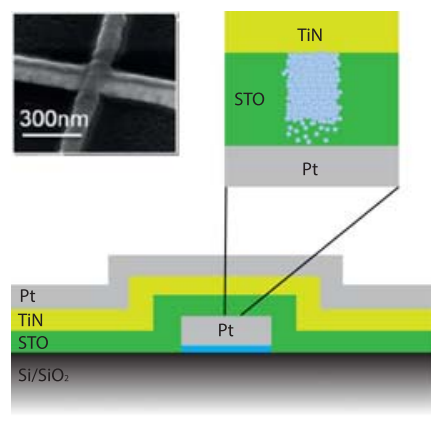

b)

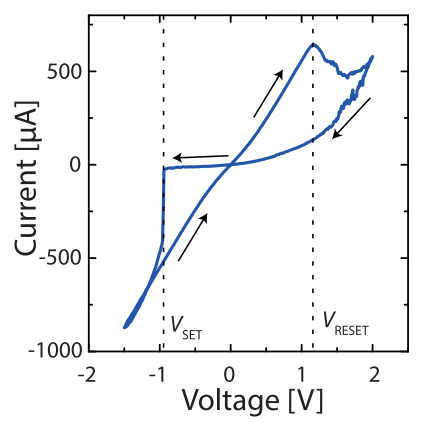

FIG. 1. (a) Layer stack of the Pt/STO/TiN devices with a sketch of the filament indicating the disc-like gap of the filament at the Pt electrode in the HRS and a scanning electron micrograph of a nano-crossbar cell. (b) Exemplary $I-V$ curve measured on the Ti-rich sample. The voltage is applied to the Pt electrode. The SET and RESET voltages are marked. 
the oxide layer and a conductive filament evolves. Filament widths as small as few nanometers have been reported. ${ }^{7}$ In asymmetric stacks with different metals used for the two electrodes, the switching is expected to occur at the Schottky interface between the filament and the electrode with the higher work function and lower oxygen affinity (in this work $\mathrm{Pt}$ ) whereas the other interface is usually neglected. ${ }^{8}$ During the SET, a negative bias at the active electrode $(\mathrm{Pt})$ attracts the positively charged oxygen vacancies, thereby lowering the Schottky barrier. Applying a positive bias pushes the vacancies away from the active interface, resulting in an insulating layer and an overall high resistance (Fig. 1(b)). For a deeper understanding of the resistive switching process it is crucial to consider the involved kinetics, especially since they feature a highly nonlinear voltage dependence, spanning over several orders of magnitude in time with only a minor change in the applied voltage. ${ }^{9-15}$ Prior publications identified Joule heating and the resulting thermal activation of oxygen vacancy hopping as the source of this nonlinearity. ${ }^{10,13-17}$ Current studies are discussing the possibility of oxygen transfer reactions between the oxide and the metal electrodes as additional effects involved in VCM-type resistive switching. ${ }^{18-20}$

Due to its thoroughly investigated defect chemistry, strontium titanate (STO) is often used as a model system for resistive switching. ${ }^{21,22}$ Recently, several studies have dealt with the influence of stoichiometry on resistive switching in STO, revealing differences in forming voltage and statistics $^{23}$ data retention, ${ }^{24}$ or even switching/no-switching. ${ }^{25}$ However, in order to draw any conclusions on relevant differences in the switching mechanisms, more detailed studies are necessary. Therefore, this work addresses the question whether there is a significant influence of the composition of STO thin films on the switching kinetics. Utilizing nanocrossbar devices with a cell area of $(100 \mathrm{~nm})^{2}$ and analyzing the transient currents during pulse switching enabled the measurement of the SET and RESET kinetics over 12 orders of magnitude in time. The study was performed for STO films of three different $\mathrm{Sr} / \mathrm{Ti}$ compositions. Their SET kinetics reveal distinctive differences regarding the absolute voltages and the degrees of nonlinearity, which are further discussed using the analytical model introduced in one of our previous papers. ${ }^{14}$ They can be explained by assuming differences in thermal conductivity and the activation energy for oxygen vacancy migration. The RESET kinetics are analyzed by two means, the dependency of the resulting HRS resistances on pulse-length and amplitude and an analysis of the transient currents following the one suggested by Marchewka et al. ${ }^{26}$ Although the resistance maps are similar, the composition has a major influence on the duration of the RESET transition.

\section{EXPERIMENTAL}

\section{A. Nano-crossbar cells}

Three STO films of about $8 \mathrm{~nm}$ thickness with cation compositions $[\mathrm{Sr}] /([\mathrm{Sr}]+[\mathrm{Ti}])$ of 0.46 (Ti-rich), 0.50 (stoichiometric), and 0.57 (Sr-rich) were grown by plasmaassisted atomic layer deposition at $350{ }^{\circ} \mathrm{C}$ on top of a $\mathrm{Pt}$ coated silicon wafer with a $\mathrm{Ti}$ adhesion layer $\left(\mathrm{Si} / \mathrm{SiO}_{2}\right.$ $(430 \mathrm{~nm}) / \mathrm{Ti}(5 \mathrm{~nm}) / \mathrm{Pt}(30 \mathrm{~nm})$ (Fig. 1(a)). Using a postdeposition rapid thermal annealing at $600{ }^{\circ} \mathrm{C}$ in nitrogen atmosphere, the STO films are crystallized. Further details on the growth process can be found in recent publications. ${ }^{23,27}$ For the use as bottom electrode, the Pt film is structured into $100 \mathrm{~nm}$ wide bars by means of nanoimprint lithography and reactive ion beam etching (RIBE). The resistive switching cell is completed by a $30 \mathrm{~nm}$ TiN film, deposited by sputtering, and capped with a Pt layer. By use of e-beam lithography and RIBE, the TiN/Pt top electrode is also structured into $100 \mathrm{~nm}$ wide bars, thus resulting in active device areas of $100 \times 100 \mathrm{~nm}^{2}$ (Fig. 1(a)).

To investigate the SET kinetics, two measurement systems are applied for the different time-scales. A Keithley 4200 SCS with a two channel pulse measurement unit with remote amplifiers is used for pulses from $100 \mathrm{~ns}$ up to $1 \mathrm{~s}$ with a maximum time resolution of $200 \mathrm{MS} / \mathrm{s}$. Channel 1 (connected to the $\mathrm{Pt}$ bottom electrode) provides the signal with channel 2 (connected to the TiN top electrode) remaining on ground potential and measuring the current. For the time scale above $0.1 \mathrm{~s}$, a Keithley $2636 \mathrm{~A}$ source meter unit is used to apply the voltage, while the internal clock of the measurement PC takes the time. The overlap of one order of magnitude for both setups ensures compatibility of the measurements. All voltages in this work are applied to the Pt bottom electrode, while the TiN electrode remains grounded.

\section{RESULTS AND DISCUSSION}

\section{A. Electroforming}

In their pristine state, all of the stoichiometric and Srrich $\mathrm{Pt} / \mathrm{STO} / \mathrm{TiN}$ devices are highly resistive with resistances above $1 \mathrm{G} \Omega$ at $0.1 \mathrm{~V}$. As indicated before, these devices need an initial electroforming step before they can be switched repeatedly. For those cells that require electroforming, a voltage ramp down to $-6 \mathrm{~V}$ with a current compliance of $10 \mu \mathrm{A}$ is applied to the Pt bottom electrode using an Agilent B1500A semiconductor analyzer. This procedure is unnecessary for about $50 \%$ of the Ti-rich cells as they are already in a low resistive state of a few $\mathrm{k} \Omega$. The cumulative distribution function of the forming voltages measured on 30 cells for each stoichiometry is depicted in Figure 2 revealing huge differences. Whereas both, the Sr-rich and the stoichiometric samples, have similar ranges of $-1.8 \mathrm{~V}$ and $-2.2 \mathrm{~V}$, their median forming voltages differ by nearly a factor of two (STO: $-5.2 \mathrm{~V}$ vs. Sr-rich: $-2.7 \mathrm{~V}$ ). The distribution of the Ti-rich sample on the other hand is quite different as it features two different slopes that point towards two different populations of cells. About $25 \%$ of the cells form between -1.1 and $-1.75 \mathrm{~V}$, which is roughly equal to typical SET voltages. So, on top of about half of the cells not needing any forming because of their initially low resistances, half of the remaining cells can be considered forming-free due to their low $V_{\text {form }}$. The other $25 \%$ of the cells need much higher voltages of up to $-5.8 \mathrm{~V}$. Ti-rich STO has a tendency towards the formation of a secondary $\mathrm{TiO}_{x}$ phase when the saturation limit for excess $\mathrm{TiO}_{2}$ of 0.5 at. $\%$ is surpassed. ${ }^{28,29}$ In one of our previous studies on films deposited under the 


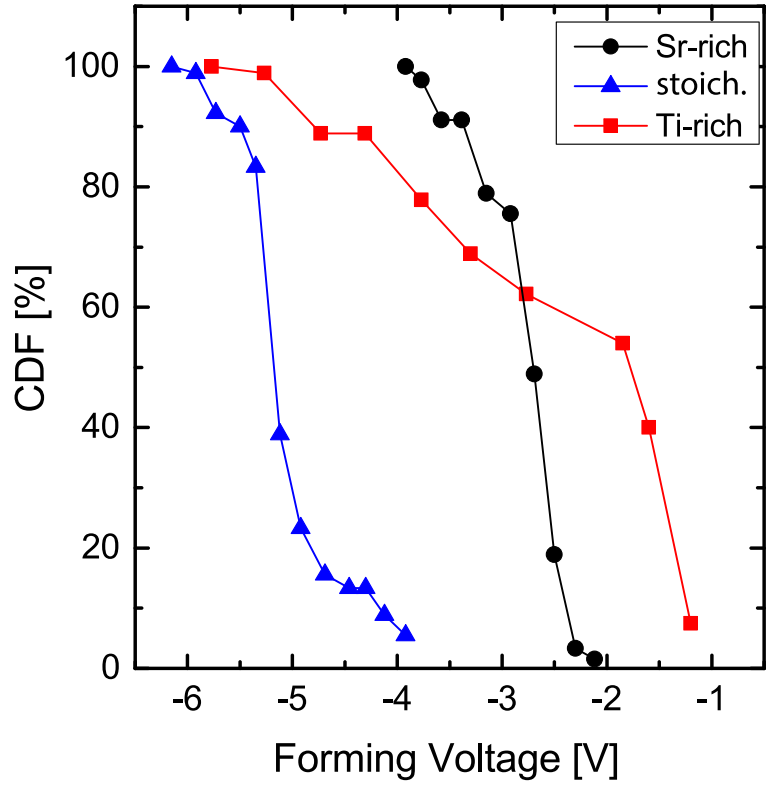

FIG. 2. Cumulative distribution functions of the forming voltages. The stoichiometric sample needs considerably higher voltages to form, whereas the Ti-rich sample shows a huge spread of the forming voltage.

same conditions, ${ }^{23}$ we demonstrated that the Ti-rich samples feature areas with different surface morphologies and significantly different local conductivities, that were interpreted as such $\mathrm{TiO}_{x}$ phases. These $\mathrm{TiO}_{x}$ regimes of about $1 \mu \mathrm{m}^{2}$ area make up around $1 / 3$ of the overall surface. These values have been extracted from conductive AFM measurements. The huge range of forming voltages and the high percentage of initially low resistive cells might be attributed to the crosspoint (i.e., $\left.(100 \mathrm{~nm})^{2}\right)$ of the cell sandwiching either a $\mathrm{TiO}_{\mathrm{x}}$-rich region or a $\mathrm{SrTiO}_{3}$ one.

\section{B. Quasi-static switching}

Figure 3(a) shows the current-voltage characteristics of a typical cell for each composition with maximum voltages of $-1.5 \mathrm{~V}$ for the SET and $2 \mathrm{~V}$ for the RESET branch. These voltage sweeps were performed using a Keithley $2636 \mathrm{~A}$ source meter with $10 \mathrm{mV}$ voltage steps and an effective sweep rate of about $4.5 \mathrm{~V} / \mathrm{s}$ starting with the negative polarity. As the cells are self-limiting, the use of a current compliance is not necessary, and not advised to ensure a better comparability with the pulse measurements, which are incompatible with a current compliance. Nonetheless a rather high current compliance of $1 \mathrm{~mA}$ is applied in the quasi static measurements to prevent damage to cells due to the application of higher currents for longer times. This current value is seldom reached during cycling, an example is the curve measured on the Sr-rich cell (black) in Figure 3(a) that enters the current compliance at about $-1.4 \mathrm{~V}$.

Overall, the $I-V$ characteristics of the three different compositions look rather similar, but there is a distinct difference in the switching voltages, with $V_{\text {SET }}$ being significantly higher $(0.2 \mathrm{~V})$ for the stoichiometric and $V_{\text {RESET }}$ being lower by about the same amount for the Sr-rich sample. This tendency is confirmed by a statistical analysis of 100 cycles on each sample (Figure 3(b)). SET and RESET voltages of the Sr-rich and stoichiometric sample are symmetric with the former requiring lower voltages $(\approx \pm 1.08 \mathrm{~V}$ compared to $\approx \pm 1.25 \mathrm{~V})$. The Ti rich sample on the other hand has asymmetric SET and RESET voltages of $V_{\mathrm{SET}}=-1.00 \mathrm{~V}$ and $V_{\text {RESET }}=1.35 \mathrm{~V}$. Overall, these results are consistent with the ones reported earlier for $12 \mathrm{~nm}$ thick STO layers. ${ }^{23}$ The lower spreading of the switching voltages for the Sr-rich devices in this study compared to the former one is attributed to the higher current compliance.

Another quite interesting difference regards the LRS branch of the $I-V$ curves (Figure 3(a)). Compared to the Tirich and the stoichiometric sample, the Sr-rich sample shows a higher nonlinearity in the LRS. As differences in the LRS, textiI-V curves have also been reported by earlier works this may point towards a difference in the conduction mechanism based on the STO composition. ${ }^{23,24}$

\section{SET kinetics}

Figure 4(a) shows the pulse scheme applied to the cells with the Keithley 4200. It starts with a $10 \mu$ s long RESET a)

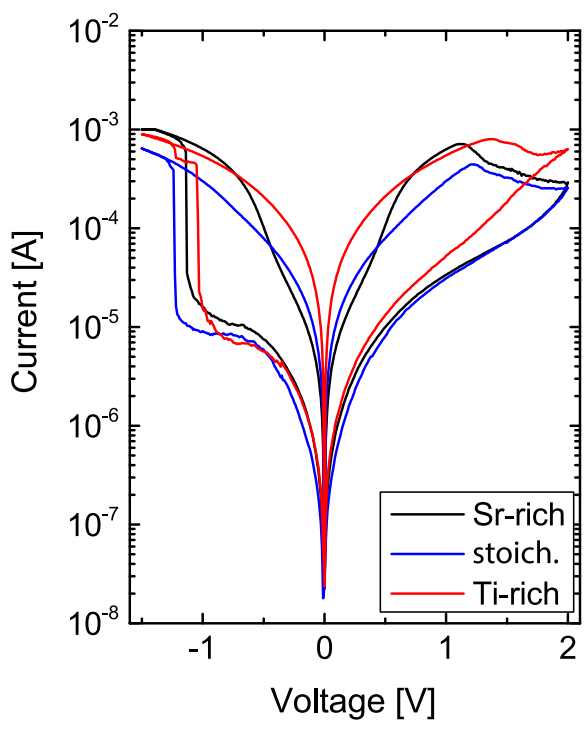

b)

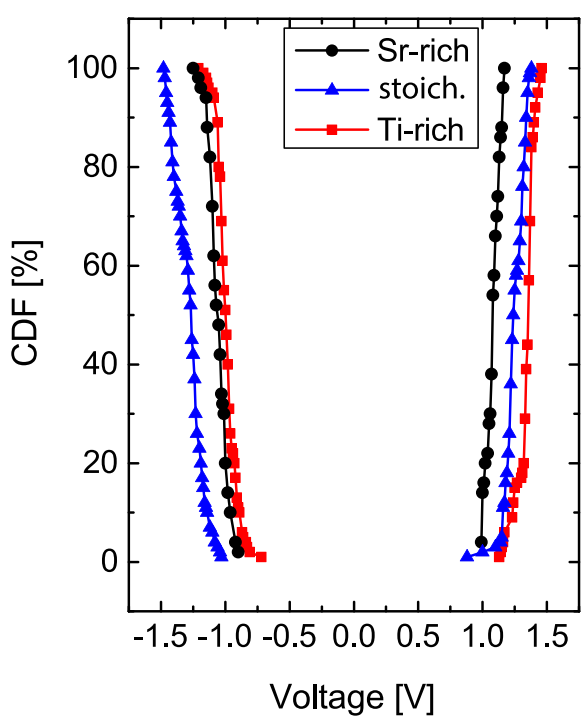

FIG. 3. (a) $I-V$ characteristic after forming. (b) Cumulative distribution function of the SET and RESET voltages taken from 100 cycles. 
a)

SET kinetics pulse scheme I:

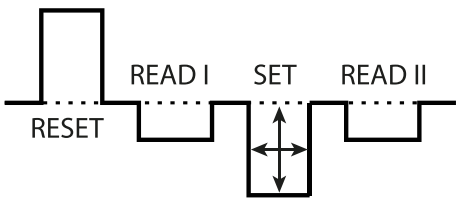

b)

SET kinetics pulse scheme II:

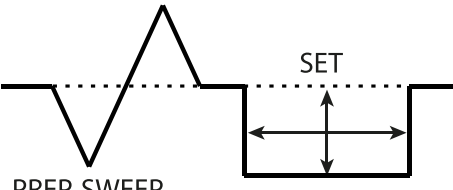

c)

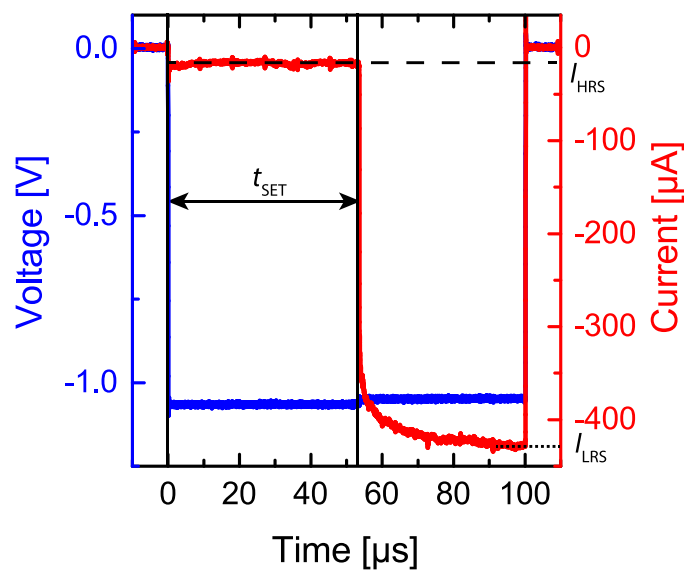

FIG. 4. (a) Pulse scheme for the fast pulse measurements. (b) Measurement scheme used for times over $0.1 \mathrm{~s}$. (c) Transient current during a $100 \mu$ s SET pulse, the SET transition occurs after $53 \mu \mathrm{s}$ as indicated by the rise in current. The HRS and LRS currents are marked by dashed and dotted lines, respectively. pulse at $2 \mathrm{~V}$ that leaves the cell in a high resistive state whose resistance is verified by a $1 \mathrm{~ms}$ long READ at $0.5 \mathrm{~V}$. The pulse width of the consecutive SET is varied between $100 \mathrm{~ns}$ and $1 \mathrm{~s}$. For each width, the amplitude is decreased in $50 \mathrm{mV}$ steps from $1.7 \mathrm{~V}$ to $0.8 \mathrm{~V}$ with each consecutive cycle. A second READ verifies the SET and completes the cycle (Fig. 4(a)). To ensure a comparable initial state every second cycle, a preparation cycle with fixed values for the SET $(-1.5 \mathrm{~V}$ for $100 \mu \mathrm{s})$ and a slightly higher RESET $(2.3 \mathrm{~V}$ for $10 \mu \mathrm{s})$ is applied.

Figure 4(b) shows the measurement scheme for the slower setup with the Keithley 2636 A. Each cycle starts with the preparation of the cell by a voltage sweep (sweeprate: $1 \mathrm{~V} / \mathrm{s}$ ) with maximum amplitudes of $-1.5 \mathrm{~V}$ and $2 \mathrm{~V}$ for the SET and RESET branch, respectively. Afterwards, a DC voltage is applied to the cell until either the SET occurs or a pre-defined time frame of $10^{4} \mathrm{~s}$ has passed. The amplitude of this DC signal is systematically lowered in $20 \mathrm{mV}$ steps until the cell was unable to SET in the given time-frame for three consecutive times.

The transient current responses of a Sr-rich cell to a $100 \mu$ s SET pulse of $-1.1 \mathrm{~V}$ is shown in Figure 4(c). For the first $53 \mu \mathrm{s}$, the current stays at a rather low level of about $-20 \mu \mathrm{A}$ in which the cell is still in the HRS. The increase in current at $53 \mu$ s marks the SET transition; for further analysis, the time between the voltage reaching $90 \%$ of its plateau value and the beginning of the SET transition is termed SET time $t_{\mathrm{SET}}$. After the SET, the current remains on a high absolute value of about $-420 \mu \mathrm{A}$ for the rest of the pulse.

The as expected highly nonlinear voltage dependency of $t_{\text {SET }}$ for the three different STO compositions is depicted in Figure 5(a). The trend for the Sr-rich sample (black) starts at an amplitude of $-0.7 \mathrm{~V}$ with $t_{\text {SET }}$ around $10^{3}$ to $10^{4} \mathrm{~s}$ and then decreases rapidly down to slightly below $10 \mathrm{~ns}$ for voltages between -1.1 and $-1.4 \mathrm{~V}$. For devices with stoichiometric STO (blue), the $t_{\text {SET }}$ vs. $V$ curve is shifted about $0.3 \mathrm{~V}$ towards higher amplitudes, consistent with the quasi-static measurements. Compared to the other compositions the Tirich STO reveals a significantly flatter slope, starting at $-0.35 \mathrm{~V}$ just below $10^{4} \mathrm{~s}$ and ending at $-1.55 \mathrm{~V}$ for $10 \mathrm{~ns}$, with a few outliers around $-1.7 \mathrm{~V}$ and $1 \mu \mathrm{s}$. For a further analysis, the analytical model proposed in one of our previous publications is used. ${ }^{14}$ Assuming an ion hopping mechanism described by the Mott-Gurney law for the oxygen vacancies, the SET time can be estimated as

$$
t_{\mathrm{SET}}=\frac{l_{\mathrm{disc}}}{v_{\mathrm{drift}}}=\frac{l_{\text {disc }}}{a f} \exp \left(\frac{\Delta W_{\mathrm{hop}}}{k_{\mathrm{B}} T}\right) \sinh \left(\frac{z e a}{2 k_{\mathrm{B}} T} \frac{V}{l_{\mathrm{disc}}}\right)^{-1} .
$$

a)

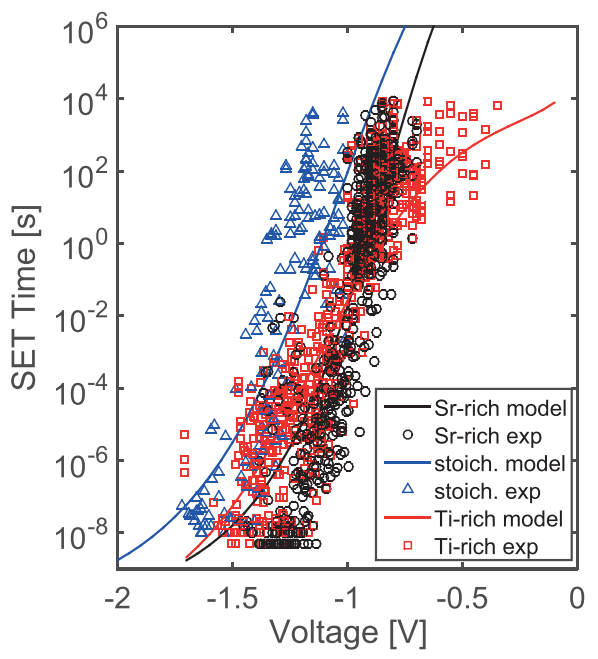

b)

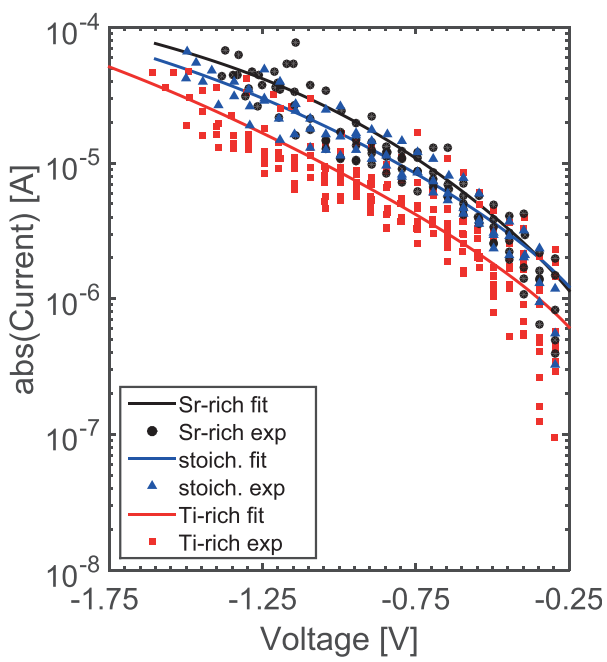

FIG. 5. (a) The SET time $t_{\mathrm{SET}}$ as a function of the applied voltage, experimental (dots), model (lines) (b) $I-V$ measured by 130 pulses with amplitudes between -0.25 and $-1.6 \mathrm{~V}$. The lines indicate the fit using Equation (3). 
The hopping mechanism is specified by the hopping distance $a$, the attempt frequency $f$, and the activation energy $\Delta W_{\text {hop }}$, where the $\sinh ()$ term describes the barrier lowering due to high electric fields in the disc-like filament gap of length $l_{\text {disc. }}{ }^{13,14}$ As mentioned earlier, the local temperature $T$ in the filament, or more precisely in the gap between the filament and the active electrode, is the main origin of the nonlinear switching kinetics. It can be approximated as

$$
T=T_{0}+R_{\mathrm{th}} \cdot V I=T_{0}+R_{\mathrm{th}} \cdot P,
$$

where $R_{\text {th }}$ is the effective thermal resistance and $T_{0}$ equals $300 \mathrm{~K}$. For an estimation of this temperature, it is essential to use an $I-V$ characteristic for a preferably large voltage range. With quasi-static sweeps, this cannot be achieved due to the relatively low SET voltages which can be overcome by the fast pulse experiments. Therefore, the method of choice is to measure the $I-V$ characteristic using a pulse scheme similar to Figure 4(a). The only changes made are to set a fixed width for the SET pulse of $100 \mu \mathrm{s}$ and a voltage range from $-0.25 \mathrm{~V}$ to $-1.6 \mathrm{~V}$. Compared to a conventional quasistatically measured $I-V$ curve, this method provides a significant advantage. Due to the much higher sampling-rate of the pulse setup, the HRS currents can be extracted from the transient currents (cf. Fig. 4) as long as $t_{\mathrm{SET}}$ is not shorter than the timeframe per sample, independent if the cell switches at the applied voltage. This allows a characterization of the HRS beyond the usual quasi-static $V_{\text {SET }}$.

Figure 5(b) shows the determined $I-V$ data sets. Each point represents the mean HRS current measured from the beginning of the pulse until either the end of the pulse or the reaching of $t_{\text {set }}$ if a SET occurred during the pulse. The resulting $I-V$ curves are fitted using a diode-like characteristic in series with a resistor: ${ }^{14,30}$

$$
I=-I_{0}+\frac{V_{0}}{R_{\mathrm{s}}} \cdot \mathrm{W}\left(\frac{I_{0} R_{\mathrm{s}}}{V_{0}} \cdot \exp \left(\frac{V+I_{0} R_{\mathrm{s}}}{V_{0}}\right)\right)
$$

with the saturation current $I_{0}$, the series resistance $R_{\mathrm{S}}$, and a characteristic voltage $V_{0}=\eta V_{\mathrm{T}}=\eta \frac{k_{\mathrm{B}} T}{e}$, including the thermal voltage $V_{\mathrm{T}}$ and a nonideality factor $\eta$. W is Lambert's W function, which is the inverse function to $f(x)=x e^{x} .{ }^{31} \mathrm{We}$ decided to use a fixed value for $V_{\mathrm{T}}$ disregarding its temperature dependence, because the fit mainly serves to give a handle for the measured $I-V$ characteristics. The parameters used for fitting the $I-V$ curves and for calculating the SET times are given in Table I. In Figure 5(a), the calculated kinetics are represented by the colored lines. A comparison to the measured data compiled from multiple cells on each sample reveals a good accordance. Besides the different input $I-V \mathrm{~s}$, only two parameters are changed for modeling the different stoichiometries. As the thermal conductivity in STO films decreases with increasing nonstoichiometry, ${ }^{32,33}$ be it $\mathrm{Ti}$ or $\mathrm{Sr}$ excess, we assumed a lower thermal resistance for the temperature-estimation in the stoichiometric film, which results in a lower $T_{\text {disc }}$. The second one is the activation energy for the vacancy hopping $\Delta W_{\text {hop. }}$. At this point, it has to be mentioned that the $\Delta W_{\text {hop }}$ values used in the fit exceed the ones measured for STO. ${ }^{34}$ Simulations by Marchewka ${ }^{35}$ suggest that this inconsistency may originate from the interrelation of drift and diffusion transport. For fitting the kinetics of the Ti-rich sample, the activation energy for the oxygen vacancy migration had to be lowered to $0.9 \mathrm{eV}$. This difference in the activation energy may indicate that it is not the STO that is switched but rather a possible $\mathrm{TiO}_{x}$-rich secondary phase.

\section{RESET kinetics}

The pulse scheme for the RESET kinetics is quite similar to the one used for the SET with the main difference of a fixed SET $(500 \mu$ s at $-1.3 \mathrm{~V})$ and a variable RESET pulse $(100 \mathrm{~ns}$ to $100 \mathrm{~s}$ at $0.5 \mathrm{~V}$ to $2 \mathrm{~V}$ ) opposed to a variable SET and a fixed RESET (cf. Fig. 6(a)). For the preparation cycle, a $100 \mu \mathrm{s}$ SET at $-1.3 \mathrm{~V}$ and a $10 \mu$ s long RESET at $2 \mathrm{~V}$ are used.

In contrast to the rather spontaneous SET event, the RESET is a more gradual process. The origin of this gradual nature of the RESET was found to be the interaction of two competing driving forces for oxygen vacancy migration, drift and diffusion, and their convergence towards an equilibrium state. ${ }^{26}$ After the SET, the oxygen vacancies are distributed rather homogenously throughout the filament. At the beginning of the RESET, oxygen vacancies are removed from the active interface by drift, thus lowering the local oxygen vacancy concentration. Due to the resulting concentration, gradient diffusion sets in, providing a driving force towards the active electrode. So, drift and diffusion tend towards different directions and the RESET process continues until both driving forces are in an equilibrium. ${ }^{26}$ The RESET transient characteristic shown in Figure 6(b) comprises transient currents of all the 1.4 V RESET pulses measured on the Ti-rich sample. It demonstrates the gradual nature of the RESET vividly. In the first $300 \mathrm{~ns}$, the current increases steadily by a few $10 \mu \mathrm{A}$ up to a maximum of $570 \mu \mathrm{A}$ and decreases afterwards asymptotically to about $100 \mu \mathrm{A}$. The time where the current passes half of the difference between the maximum and minimum value is called $\tau_{50}$. A similar hump in the transient current was reported by Marchewka. Its origin is the redistribution of a slight oxygen vacancy enrichment at the active interface followed by depletion and build up of an

TABLE I. Fitting parameters.

\begin{tabular}{lcccccccc}
\hline \hline & $I_{0}(\mu \mathrm{A})$ & $\eta V_{\mathrm{T}}(\mathrm{V})$ & $R_{\mathrm{S}}(\Omega)$ & $R_{\mathrm{th}}(\mathrm{k} / \mathrm{W})$ & $l_{\text {disc }}(\mathrm{nm})$ & $a(\mathrm{~nm})$ & $f(\mathrm{THz})$ & $W_{\mathrm{hop}}(\mathrm{eV})$ \\
\hline Sr-rich & 0.64 & 0.237 & 6119 & $15 \times 10^{6}$ & 4 & 0.2 & 16.67 \\
STO & 1.16 & 0.339 & 4561 & $10 \times 10^{6}$ & 4 & 0.2 & 16.67 \\
Ti-rich & 0.62 & 0.362 & 2930 & $15 \times 10^{6}$ & 4 & 0.2 & 16.67 \\
\hline \hline
\end{tabular}


a)

RESET kinetics pulse scheme:

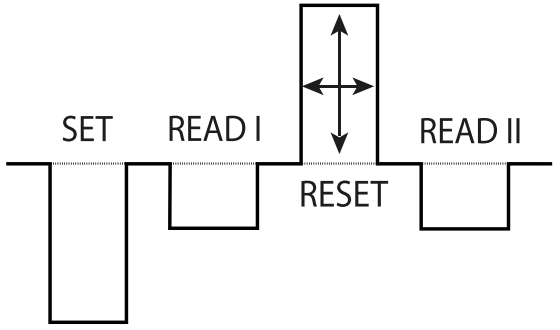

b)

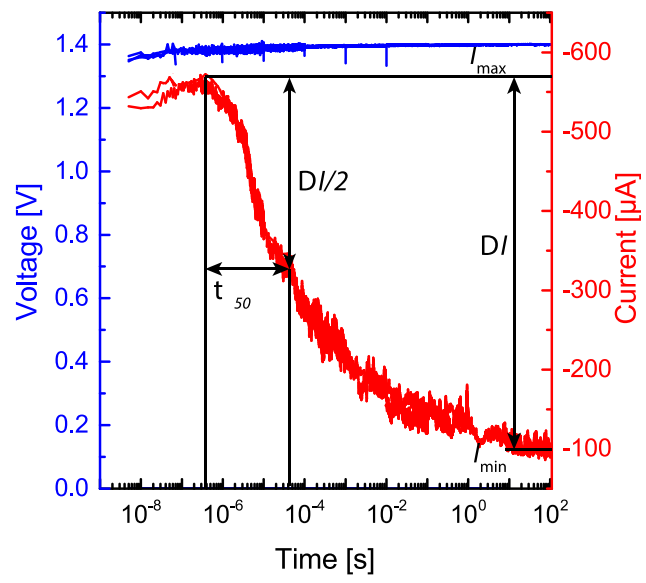

FIG. 6. (a) Pulse scheme for measuring the RESET kinetics. (b) Combined transient current of all $1.4 \mathrm{~V}$ RESET pulses on the Ti-rich sample. The RESET transition is gradual in nature, with the largest change in resistance taking place right after the pulse plateau has been reached. oxygen vacancy gradient. $^{26}$ Figures $7(a)-7(c)$ show the dependencies of the resistance after the RESET (READ II in Figure 7) on the width and amplitude of the applied RESET pulse for an exemplary cell of each composition. Note that the same color scale for the cell resistance is used in all three plots. At the first glance, these $V-t-R$ plots look rather similar in shape for the three different stoichiometries. The down left half is dark blue in color, indicating a low resistance as pulse length and/or amplitude are not sufficient to RESET the cell into the HRS. Then, there is a transitional area, indicated by a lighter blue, where the cell resistance is slightly increased (lower $10 \mathrm{k} \Omega$ regime) by the RESET pulse but not yet fully switched. The third region, located in the upper right corner, shows the highest change in resistance. It is this region where differences in the RESET kinetics for the different compositions are revealed. Whereas the resistance of the Ti-rich cell seldom exceeds $100 \mathrm{k} \Omega$, the $\mathrm{Sr}$-rich and the stoichiometric cell reach resistances well above. From this simple discussion on the resistance values of view, one might suspect that the RESET is slower for the Ti-rich cell, because of the overall lower HRS. But taking the transient currents into account and analyzing the respective $\tau_{50}$ values, this impression is put into another perspective. In Figure 8, the combined RESET transients (cf. Fig. 6(b)) for all $1.7 \mathrm{~V}$ RESET pulses are plotted for all three compositions. The

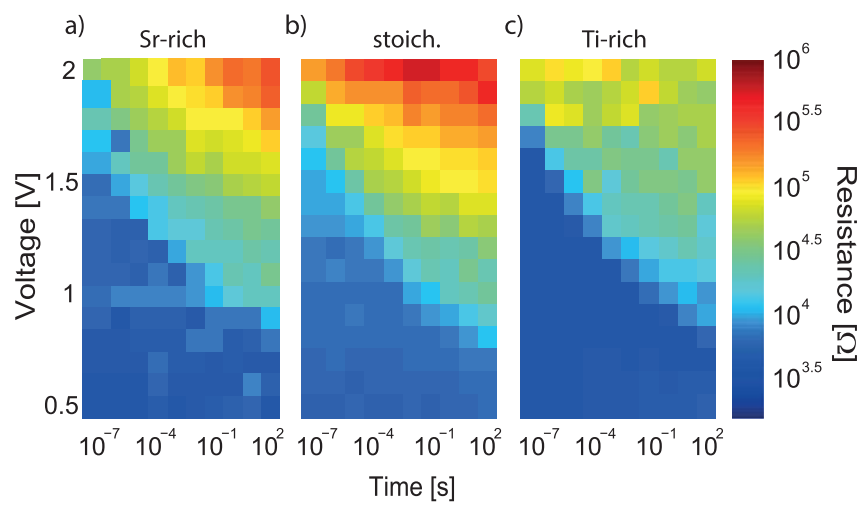

FIG. 7. Resistance after the RESET as a function of the applied voltage and the pulse-length for (a) the Sr-rich, (b) the stoichiometric, and (c) the Ti-rich STO sample. resulting $I-t$ characteristics reveal significant differences in the dynamics of the RESET process. One being the different starting LRS currents, which fit well to the quasi-static $I-V$ measurements (cf. Fig. 3). Another one being the differing

a)

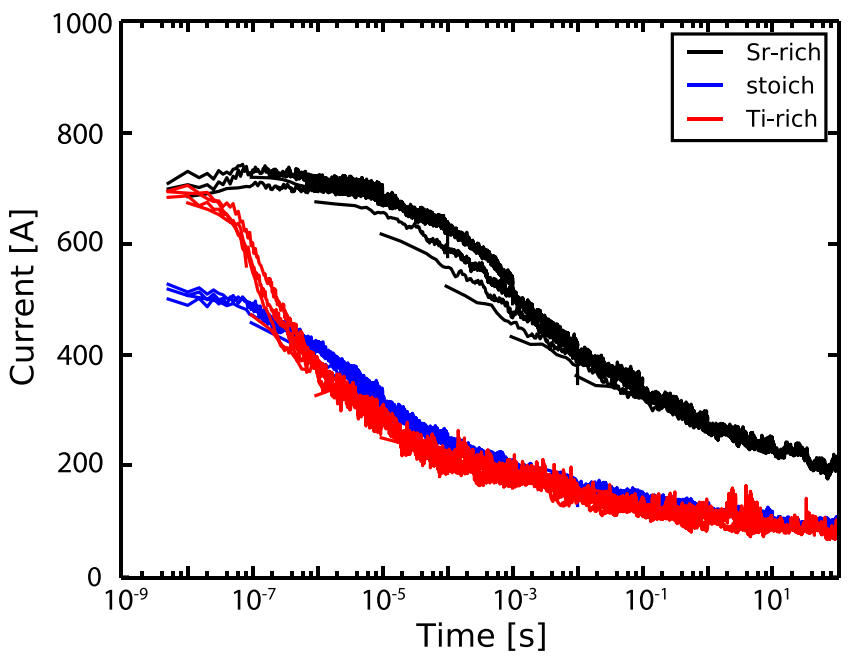

d)

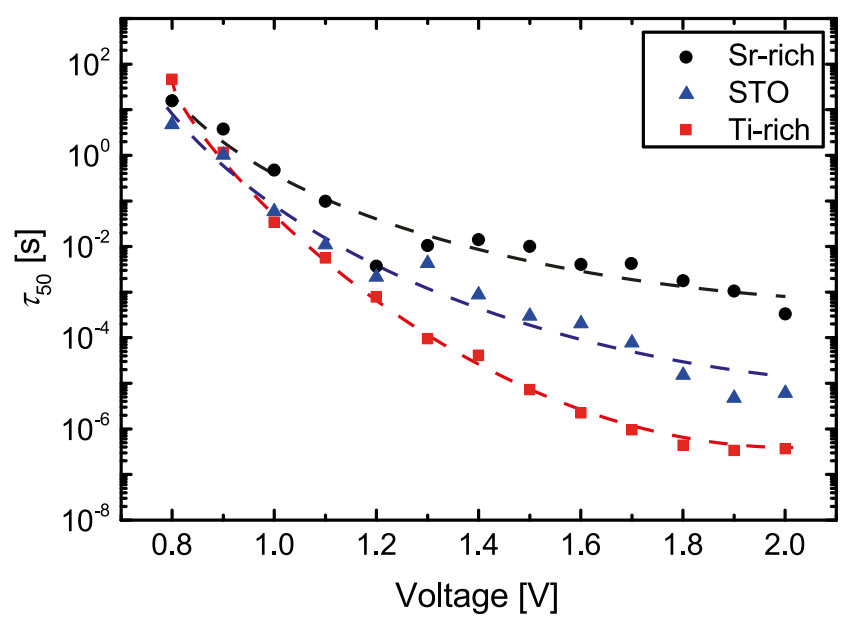

FIG. 8. (a) The combined transient currents at a RESET voltage of $1.7 \mathrm{~V}$ reveal significant differences in the RESET kinetics for the three compositions. (b) The decay time $\tau_{50}$ for different voltages display the difference in pace of the RESET process. 
paces of the RESET process for the different compositions. For the Sr-rich sample, the current decrease that accompanies the RESET features a prominent hump and occurs much slower, especially compared to the Ti-rich sample. These differences in the dynamics of the transient currents during the RESET can be summarized by the $\tau_{50}$ values. In Figure 8(b), the $\tau_{50}$ vs. voltage graphs for each composition are shown. For low voltages $(<1.0 \mathrm{~V})$, the difference in the decay times for the various compositions is rather small as all $\tau_{50}$ values are around a few seconds. It is this regime of time and voltage where the quasi-static measurements (cf. Fig. 3) are made. The small difference in time is consistent with the small difference of the observed $V_{\text {RESET }}$. With increasing voltage, the three curves diverge with the Sr-rich sample featuring the highest and the Ti-rich sample the smallest $\tau_{50}$. For the highest voltages applied to cells $(>1.8 \mathrm{~V})$, the $\tau_{50}$ values differ by more than three decades. Simulations by Marchewka $^{36}$ show that variations in the activation energy $\Delta W_{\text {hop }}$ of a few $0.1 \mathrm{eV}$ can explain why the device with the lower $\Delta W_{\text {hop }}$ switches several orders of magnitude faster. This again points towards a lower activation energy for the Ti-rich sample. In contrast, a possible explanation for the RESET being faster in the stoichiometric compared to the Sr-rich sample may be given by a lower temperature in the filament due to a lower LRS current and a higher thermal conductivity. As diffusion works towards an even distribution of oxygen vacancies throughout the filament, it slows down the RESET process. An overall higher temperature facilitates diffusion as described by the Einstein relation

$$
D(T)=\mu(T) \cdot k_{\mathrm{B}} \cdot T
$$

with $D(T)$ being the diffusion coefficient and $\mu(T) \propto 1 / T$ $\cdot \exp \left(\Delta W_{\text {hop }} / k_{\mathrm{B}} \cdot T\right)$ the oxygen vacancy mobility. Therefore, higher temperatures favor diffusion over drift, which may explain the slower RESET in the Sr-rich sample. Also, the prominent hump in the RESET transient of the Sr-rich sample suggests a higher accumulation of oxygen vacancies at the active interface that have to be moved, which also matches the higher LRS current in the Sr-rich sample compared to the stoichiometric one. The redistribution of such an accumulation at the beginning of the RESET might also be an explanation for the observed differences between the $\mathrm{Sr}$ rich and the stoichiometric device.

\section{E. Retention}

Before measuring the retention, the cells are prepared in either the LRS or the HRS by a sweep to $-1.5 \mathrm{~V}$ or $2 \mathrm{~V}$, respectively. For both resistive states, the retention is measured by applying a $2.5 \mathrm{~ms}$ READ at $-0.3 \mathrm{~V}$ in logarithmically increasing intervals up to a total time of $10 \mathrm{~h}$. The ambient temperature is increased to $100{ }^{\circ} \mathrm{C}$ for a temperature acceleration of the states' lifetimes. From Eqs. (1) and (2) at an increased ambient temperature $T_{0}$ of $373 \mathrm{~K}$ and a READ voltage of $-0.3 \mathrm{~V}$, one would expect the Ti-rich cell to SET after about $1 \mathrm{~s}$. The samples of the other two compositions should not even switch until about $10^{3} \mathrm{~s}$. Both estimated times are longer than the cumulated time of all READs of $0.175 \mathrm{~ms}$. Therefore, no disturbance by the READ is expected.

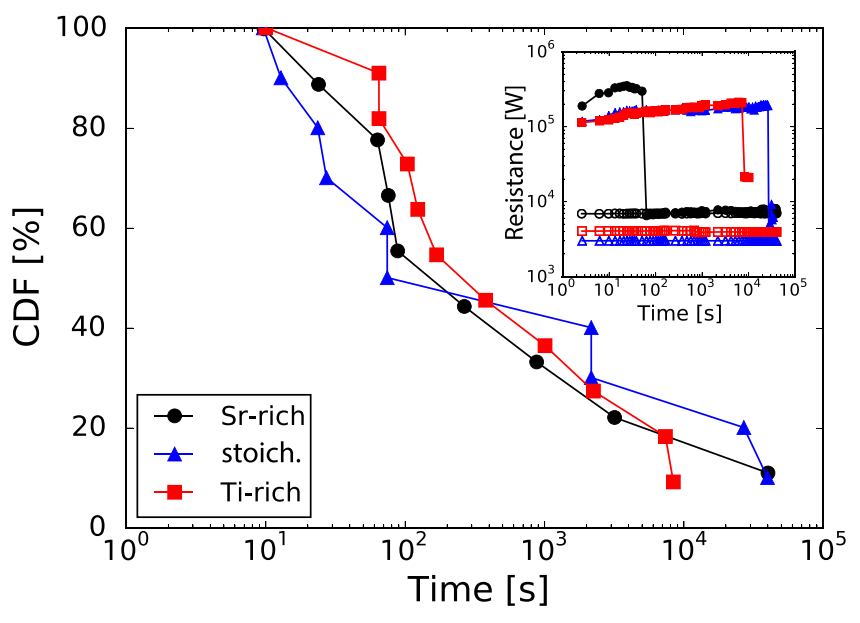

FIG. 9. (a) HRS/LRS resistances plotted versus the pass time for the Sr-rich (blue), stoichiometric (black), and Ti-rich (red) samples. (b) Cumulative distribution function of the HRS lifetimes measured at $100^{\circ} \mathrm{C}$.

The inset of Figure 9 shows exemplary resistance vs. time curves for each stoichiometry. While the LRS are stable, the HRS decay into the LRS ones. Figure 9 shows the distribution of the HRS lifetimes of the probed devices. Due to the small batch size, no statistical statement can be made, but nonetheless, it becomes clear that there is a large spread in the HRS lifetimes for all compositions. An influence of the stoichiometry on the retention could not be found which is surprising regarding the differences in the SET kinetics. Together with the surprising lack of any materials trend mentioned above, this points towards another effect than drift during the READ as origin of the retention failure. Most probably, this could be a relaxation of the oxygen vacancy concentration inside the filament by means of diffusion towards the Ptelectrode as the stable state is the more homogeneous one, in this case the LRS. ${ }^{35,37}$

\section{SUMMARY AND CONCLUSION}

In this paper, we presented a study of the switching kinetics for the SET and RESET process in resistive switching $\mathrm{Pt} / \mathrm{Sr}_{1+x} \mathrm{Ti}_{1+y} \mathrm{O}_{3+(x+2 y)} / \mathrm{TiN}$ nanocrossbar devices of three different $[\mathrm{Sr}] /([\mathrm{Sr}]+[\mathrm{Ti}])$ ratios. It is found that the composition of the film has a major influence on both processes.

Analysis of the electroforming revealed a major shift of $V_{\text {form }}$ depending on the stoichiometry with very large deviations for the Ti-rich film that may originate from the phase separation known to occur for high Ti concentrations in STO. The quasi-static switching curves measured after forming showed certain differences in the shape and also in the SET and RESET voltages between the different STO compositions.

The SET kinetics were studied over 12 orders of magnitude between $10 \mathrm{~ns}$ and $10^{4} \mathrm{~s}$. By analyzing the transient currents measured during pulse experiments, the SET times are extracted and their voltage dependencies investigated. With respect to the stoichiometric STO, the $t_{\mathrm{SET}}$ vs. $V$ curves of both non-stoichiometric compositions are shifted towards 
lower amplitudes. The origin of this shift may lie in the higher thermal conductivity reported for stoichiometric STO that would lead to lower cell temperatures. A comparison to an analytical model supports this hypothesis. Whereas the $t_{\mathrm{SET}}$ vs. $V$ curves of the stoichiometric and the Sr-rich samples are nearly parallel to each other, the Ti-rich sample features a flatter slope crossing the curve of the Sr-rich sample. By assuming a lower activation energy for the oxygen vacancy migration, the slope of the Ti-rich sample can also be modeled, which might indicate that the switching taking place in a $\mathrm{TiO}_{x}$ phase. For studying the RESET kinetics, a pulse series was applied and the resistances before and after the RESET were measured. The resulting resistance maps for the different compositions show no major difference beside a tendency of the Ti-rich sample towards lower HRS resistances. This conformance regarding the resistances is contrasted by a significant difference in the dynamics of the transient current and the $\tau_{50}$ values extracted therefrom. At higher RESET voltages, a clear composition dependency becomes observable with the Ti-rich STO sample switching faster and the Sr-rich STO sample switching slower than the stoichiometric STO. This tendency can be motivated by the assumption of a lower activation energy $\Delta W_{\text {hop }}$ involved in the hopping process of oxygen vacancies for the Ti-rich and an overall lower temperature in the stoichiometric sample due to lower currents and a higher thermal conductivity. These assumptions match with the ones used for fitting the SET kinetics.

By measuring the retention behavior at $100^{\circ} \mathrm{C}$, it could be shown that the HRS is less stable than the LRS and that the retention failure in the HRS does not originate from the drift during the READ process. The most reasonable mechanism is diffusion of oxygen vacancies towards the active interface to reach a more homogenous oxygen vacancy distribution inside the filament.

Based on our study, we can conclude that the properties of an ReRAM cell can be tuned by modifying the oxide layer with respect to its thermal properties. A lower thermal conductivity makes the Joule heating more effective, which may result in faster switching. Moreover, the migration enthalpy could be varied, which leads to a change in the nonlinearity of the switching kinetics and potentially to engineered retention properties. The variation of the cation (here $\mathrm{Sr} / \mathrm{Ti}$ ) ratio is a viable method to engineer the film properties of ternary metal oxides. For binary oxides doping might be a viable way. In literature, it was already demonstrated that doping $\mathrm{HfO}_{2}$ with different valent metals can influence the forming voltage. ${ }^{38,39}$ Moreover, it was shown theoretically that replacing Ti in STO can influence the effective migration barrier, too. ${ }^{40}$

This study also demonstrates that an analysis of the switching kinetics over a broad time range may reveal important additional information compared to DC characterizations or pulse studies focussed on a smaller timeframe.

\section{ACKNOWLEDGMENTS}

This work was supported by the Deutsche Forschungsgemeinschaft under Grant No. SFB 917 and also in part by the European Community's Seventh Framework Programme under ENHANCE-238409.
The authors would like to thank L. Hellmich for lab support.

${ }^{1}$ A. Chen, in Proceedings of ESSDERC (2015), Vol. 109.

${ }^{2}$ J. S. Meena, S. M. Sze, U. Chand, and T.-Y. Tseng, Nanoscale Res. Lett. 9, 526 (2014).

${ }^{3}$ R. Waser, R. Dittmann, G. Staikov, and K. Szot, Adv. Mater. 21, 2632 (2009).

${ }^{4}$ H. Akinaga and H. Shima, Proc. IEEE 98, 2237 (2010).

${ }^{5}$ S. Yu, B. Gao, Z. Fang, H. Yu, J. Kang, and H.-S. P. Wong, Adv. Mater. 25, 1774 (2013)

${ }^{6}$ R. Waser and M. Aono, Nat. Mater. 6, 833 (2007).

${ }^{7}$ D.-H. Kwon, K. M. Kim, J. H. Jang, J. M. Jeon, M. H. Lee, G. H. Kim, X.S. Li, G.-S. Park, B. Lee, S. Han, M. Kim, and C. S. Hwang, Nat. Nanotechnol. 5, 148 (2010)

${ }^{8}$ R. Waser, R. Bruchhaus, and S. Menzel, in Nanoelectronics and Information Technology, 3rd ed., edited by R. Waser (Wiley-VCH, 2012), pp. 683-710.

${ }^{9}$ S. Menzel, M. Salinga, U. Böttger, and M. Wimmer, Adv. Funct. Mater. 25, 6306-6325 (2015).

${ }^{10}$ D. Ielmini, F. Nardi, and S. Balatti, IEEE Trans. Electron Devices 59, 2049 (2012).

${ }^{11}$ T. Diokh, E. Le-Roux, S. Jeannot, C. Cagli, V. Jousseaume, J. F. Nodin, M. Gros-Jean, C. Gaumer, M. Mellier, J. Cluzel, C. Carabasse, P. Candelier, and B. D. Salvo, Thin Solid Films 533, 24 (2013).

${ }^{12}$ S. Yu, Y. Wu, and H. Wong, Appl. Phys. Lett. 98, 103514/1 (2011).

${ }^{13}$ S. Menzel, M. Waters, A. Marchewka, U. Böttger, R. Dittmann, and R. Waser, Adv. Funct. Mater. 21, 4487 (2011).

${ }^{14}$ K. Fleck, U. Böttger, R. Waser, and S. Menzel, IEEE Electron Device Lett. 35, 924 (2014).

${ }^{15}$ K. Fleck, U. Böttger, R. Waser, and S. Menzel, Mater. Res. Soc. Symp. Proc. 1790, 7 (2015).

${ }^{16}$ Y. Nishi, S. Menzel, K. Fleck, U. Boettger, and R. Waser, IEEE Electron Device Lett. 35, 259 (2013).

${ }^{17}$ P. Huang, Y. Wang, H. Li, B. Gao, B. Chen, F. Zhang, L. Zeng, G. Du, J. Kang, and X. Liu, IEEE Trans. Nanotechnol. 13, 1127 (2014).

${ }^{18}$ W. Kim, S. Menzel, D. J. Wouters, Y. Guo, J. Robertson, B. Rösgen, R. Waser, and V. Rana, Nanoscale 8, 17774 (2016).

${ }^{19}$ C. Baeumer, C. Schmitz, A. Marchewka, D. N. Mueller, R. Valenta, J. Hackl, N. Raab, S. P. Rogers, M. I. Khan, S. Nemsak, M. Shim, S. Menzel, C. M. Schneider, R. Waser, and R. Dittmann, Nat. Commun. 7, 12398 (2016).

${ }^{20}$ D. Cooper, C. Baeumer, N. Bernier, C. L. Torre, A. Marchewka, R. Dunin-Borkowski, S. Menzel, R. Waser, and R. Dittmann, "Resolving anomalous resistance hysteresis in oxide RERAM: oxygen evolution and reincorporation revealed by in-situ TEM" (unpublished).

${ }^{21}$ R. Dittmann, R. Muenstermann, I. Krug, D. Park, T. Menke, J. Mayer, A. Besmehn, F. Kronast, C. M. Schneider, and R. Waser, Proc. IEEE 100, 1979 (2012).

${ }^{22}$ C. Baeumer, N. Raab, T. Menke, C. Schmitz, R. D. Rosezin, P. Mller, M. Andr, V. Feyer, R. Bruchhaus, F. Gunkel, C. M. Schneider, R. Waser, and R. Dittmann, Nanoscale 8, 13967 (2016).

${ }^{23}$ N. Aslam, V. Longo, C. Rodenbuecher, F. Roozeboom, W. M. M. Kessels, K. Szot, R. Waser, and S. Hoffmann-Eifert, J. Appl. Phys. 116, 064503/1 (2014).

${ }^{24}$ N. Raab, C. Baeumer, and R. Dittmann, AIP Adv. 5, 047150 (2015).

${ }^{25}$ E. Mikheev, J. Hwang, A. P. Kajdos, A. J. Hauser, and S. Stemmer, Sci. Rep. 5, 11079 (2015).

${ }^{26}$ A. Marchewka, B. Roesgen, K. Skaja, H. Du, C. Jia, J. Mayer, V. Rana, R. Waser, and S. Menzel, Adv. Electron. Mater. 2, 1500233 (2016).

${ }^{27}$ V. Longo, N. Leick, F. Roozeboom, and W. M. M. Kessels, ECS J. Solid State Sci. Technol. 2, N15 (2013).

${ }^{28}$ S. Witek, D. M. Smyth, and H. Pickup, J. Am. Ceram. Soc. 67, 372 (1984).

${ }^{29}$ T. Suzuki, Y. Nishi, and M. Fujimoto, Philos. Mag. A 80, 621 (2000).

${ }^{30}$ A. Ortiz-Conde, F. Sanchez, and J. Muci, Solid-State Electron. 44, 1861 (2000).

${ }^{31}$ R. Corless, G. Gonnet, D. Hare, D. Jeffrey, and D. Knuth, Adv. Comput. Math. 5, 329 (1996).

${ }^{32}$ C. M. Brooks, R. B. Wilson, A. Schaefer, J. A. Mundy, M. E. Holtz, D. A. Muller, J. Schubert, D. G. Cahill, and D. G. Schlom, Appl. Phys. Lett. 107, 051902/1 (2015). 
${ }^{33}$ E. Breckenfeld, R. Wilson, J. Karthik, A. R. Damodaran, D. G. Cahill, and L. W. Martin, Chem. Mater. 24, 331 (2012).

${ }^{34}$ R. A. De Souza, Adv. Funct. Mater. 25, 6326-6340 (2015)

${ }^{35}$ A. Marchewka, R. Waser, and S. Menzel, in 2015 International Conference On Simulation of Semiconductor Processes and Devices (SISPAD), 9-11 September, Washington D.C., USA (2015), pp. 297-300.

${ }^{36}$ A. Marchewka, R. Waser, and S. Menzel, 2015 International Conference On Simulation of Semiconductor Processes and Devices (SISPAD), 6-8 September, Nuremberg, Germany (2016).
${ }^{37}$ M. Noman, W. Jiang, P. A. Salvador, M. Skowronski, and J. A. Bain, Appl. Phys. A: Mater. Sci. Process. 102, 877 (2011).

${ }^{38}$ L. Zhao, S.-W. Ryu, A. Hazeghi, D. Duncan, B. Magyari-Kope, and Y. Nishi, in Proceedings of the 2013 Symposium on VLSI Technology (2013).

${ }^{39}$ L. Zhao, S. Clima, B. Magyari-Kope, M. Jurczak, and Y. Nishi, Appl. Phys. Lett. 107, 013504/1 (2015).

${ }^{40}$ M. Schie, R. Waser, and R. A. D. Souza, J. Phys. Chem. C 118, 15185 (2014). 